\title{
Diacronie
}

Studi di Storia Contemporanea

$N^{\circ} 12,4 \mid 2012$

Sulle tracce delle idee

\section{Bernard Lewis, Le molte identità del Medio Oriente}

\section{Luca Zuccolo}

\section{(2) OpenEdition}

\section{Journals}

\section{Edizione digitale}

URL: http://journals.openedition.org/diacronie/2436

DOI: $10.4000 /$ diacronie. 2436

ISSN: 2038-0925

\section{Editore}

Association culturelle Diacronie

Notizia bibliografica digitale

Luca Zuccolo, « Bernard Lewis, Le molte identità del Medio Oriente », Diacronie [Online], № 12, 4 | 2012, documento 15, Messo online il 29 décembre 2012, consultato il 24 septembre 2020. URL : http:// journals.openedition.org/diacronie/2436; DOI : https://doi.org/10.4000/diacronie.2436 


\title{
Diacronie
}

\section{RECENSIONE:}

\section{Bernard LEWIS, Le molte identità del Medio Oriente, Bologna, Il Mulino, 2011, 160 pp.}

\author{
a cura di Luca ZUCCOLO *
}

Questo libro di Bernard Lewis si può descrivere con una sola frase: anche le differenze ci identificano. In questo concetto, infatti, sono racchiuse tutte le molteplici sfaccettature di un saggio redatto per la prima volta nel 1998 risultato della raccolta e ampliamento di tre paper presentati rispettivamente ai convegni di Wolfenbüttel (1989), Roma (1993) e Castegandolfo (1995) ${ }^{1}$. L'idea di questo saggio, come ribadisce lo stesso autore all'inizio dell'introduzione, è quella di esprimere «un'idea della complessità e della varietà delle identità che i gruppi, ancor più che gli individui, possono adottare in uno stesso momento; del cambiamento e dell'evoluzione costanti dell'identità mediorientale, dei modi in cui i popoli della regione percepiscono se stessi, i gruppi ai quali appartengono e le differenze tra sé e gli altri»².

Instaurando uno stretto dialogo con l'Europa, l'Occidente, le sue forme discorsive e le sue ideologie, Bernard Lewis descrive le identità mediorientali sottolineandone la pluralità e la profondità troppo spesso limitata da concetti occidentali come quelli di Patria, Nazione, lingua ed etnia:

\footnotetext{
le identità primarie sono quelle acquisite alla nascita e sono di tre tipi. La prima, segue la linea del sangue e corrisponde in ordine ascendente alla famiglia, al clan, alla tribù, fino ad arrivare alla nazione etnica. La seconda è basata sul luogo e spesso ma non necessariamente coincide con la prima, ponendosi anzi talvolta in conflitto con essa: ci si riferisce al villaggio, al vicinato, al quartiere, al distretto, alla città o alla provincia, per arrivare in tempi moderni fino allo stato. la terza, spesso intrecciata alla prima o alla seconda o a entrambe, è la comunità religiosa che può essere frammentata in sette; per molti la religione è la sola appartenenza in grado
}

\footnotetext{
${ }^{1}$ LEWIS, Bernard Le molte identità del Medio Oriente, Bologna, Il Mulino, 2011, p. 7.

2 Ibidem, p. 9.
} 
di trascendere i legami locali e immediati. Altra grande categoria dell’identità è quella che ruota attorno alla fedeltà a un governante [...]: il capo dello stato o di una branca dell'amministrazione, il governatore di una provincia o di una città, l'amministratore di un distretto, il capo villaggio.

In gran parte del mondo e per quasi tutta la storia del Medio Oriente non sono esistite altre identità al di fuori di queste due, quella involontaria della nascita e quella obbligatoria dello stato. In epoca moderna, sotto l'influenza dell'occidente, un nuovo genere di identità si sta evolvendo tra queste, vale a dire l'adesione a quelle libere forme di associazione che nel loro insieme costituiscono quella che oggi è chiamata società civile3.

Partendo da questi presupposti e dalla consapevolezza della pluralità delle identità mediorientali, nonché della loro diversità rispetto a quelle occidentali - nonostante l'adozione di un linguaggio comune -, Lewis traccia delle linee analitiche molto chiare per permettere al vasto pubblico come ai cultori della materia di approfondire la conoscenza della realtà mediorientale.

Innanzitutto, incomincia dalle "definizioni", sottolineando come i concetti occidentali, sebbene siano oramai parte del discorso politico mediorientale, sono interpretati in modo del tutto diverso dalle società e dai governi del Medio Oriente in base alle loro peculiarità intrinseche e al loro sviluppo storico politico. In questo primo capitolo, ponendo molta attenzione alle parole usate e ai loro significati, Lewis cerca di dimostrare la diversa interpretazione di termini, idee e concetti quali cittadinanza, identità, Patria e Nazione.

Il secondo capitolo, pur mantenendo una struttura similare, si concentra, invece, sulla religione. In queste pagine confrontando le tre religioni del libro e le loro rappresentazioni identitarie l'autore testimonia la pluralità e la fluidità di una società che a lungo - e ancora oggi - basa il suo concetto di identità sul fattore religioso. Ampio spazio viene dato alla religione islamica, la quale per la gran parte della storia musulmana rappresenta la definizione primaria e fondamentale dell'identità 4 . Infatti, mentre «nell'occidente laico moderno e nelle regioni che hanno accolto il punto di vista occidentale il mondo è diviso in nazioni e la nazione può essere suddivisa in diverse comunità religiose», «nella percezione musulmana [...] il mondo è diviso in religioni e queste a loro volta possono essere suddivise in nazioni e, per corruzione, in stati»5.

\footnotetext{
3 Ibidem, pp. 12-13.

4 Ibidem, p. 38.

5 Ibidem.
} 
Contrariamente alla religione, che ancora oggi riveste un ruolo decisivo nella definizione delle identità mediorientale, razza e lingua sono meno caratterizzanti, benchè, a causa dell'adesione alle categorie occidentali, stiano penetrando sempre più a fondo nella mentalità mediorientale, con notevoli contraccolpi socio-politici, descritti con chiarezza da Lewis. Gli esempi addotti dal'autore, infatti, consentono di approfondire con dati concreti argomenti teorici. Il dato fondamentale che emerge da questo terzo capitolo è la rappresentazione del Medio Oriente come un crogiuolo di lingue ed etnie difficilmente distinguibili perché reciprocamente interconnesse e modificatesi costantemente per effetto di molteplici influssi.

Di tenore ben diverso sono i capitoli seguenti, in cui Lewis descrive, definisce e spiega tre temi cardine per capire il Medio Oriente moderno e contemporaneo: la Patria (capitolo IV), la Nazione (capitolo V) e lo Stato (capitolo VI).

Viene qui dimostrato come il concetto di patria fosse già presente nella regione, seppur declinato in modo dissimile da quanto avveniva in occidente, e si sia poi evoluto - grazie alla all'influsso delle ideologie occidentali - in qualcosa di molto simile al concetto di Patria a cui siamo abituati; viene spiegato come le categorie occidentali abbiano fatto evolvere le "patrie" orientali in Nazioni e come questo abbia sconvolto la geografia e la geografia umana dell'intera regione; infine viene affrontato il culmine di questo processo di definizione dell'identità: lo Stato.

Il capitolo dedicato allo Stato e alla sua evoluzione come fonte di identità è, infatti, il più stimolante ed interessante per la definizione e la comprensione del Medio Oriente contemporaneo e futuro. Il legame con lo Stato risale e permane fin dalle origini delle formazioni socio-politiche della regione. Accanto - e forse più della religione - è lo Stato e l'appartenenza ad esso, tramite la fedeltà al regnate/governo di turno, che stabilisce il carattere identitario più significativo dei popoli del Medio Oriente. Esempio chiarificatore è la Turchia contemporanea, che unisce una fedeltà incrollabile nello Stato laico ad un'altrettanto incrollabile fedeltà nel suo esercito, braccio armato del potere e primo artefice della sopravvivenza dello Stato.

Centrale in questi tre capitoli, tuttavia, non è solo il ruolo dello Stato, ma anche le sue modificazioni storiche e materiali dovute agli influssi esogeni, particolarmente europei. Proprio queste influenze hanno imposto - nel corso della lunga storia del Medio Oriente - modelli e categorie politico-sociali molto rigide, quali quelle di nazione e nazionalismo, che non sono ancora state completamente "digerite".

Ancora una volta il caso ottomano ci viene in aiuto, come sottolinea a più riprese Lewis, dimostrando come le società mediorientali, nonostante i forti influssi e le pressioni occidentali, siano state in grado di elaborare un discorso politico del tutto 
autonomo ed innovativo, rispondente alle sfide imposte loro dalla modernità e dalle condizioni sociali endogene.

Completamento di questa sezione è il capitolo VII sul simbolismo, in cui con pochi cenni viene descritta la penetrazione di molti simboli occidentali, quali la bandiera o l'inno nazionale, nelle società mediorientali e come alcuni simboli tradizionali si siano modificati oppure ne siano stati creati di completamente nuovi. Qui più che altrove si coglie il peso della dialettica con Benedict Anderson e il suo Le Comunità immaginate, soprattutto quando Lewis si sofferma sull'invenzione delle nuove bandiere nazionali o dei simboli patriottici come la mezzaluna.

L'ottavo capitolo, invece, tocca l'ultimo punto della definizione dell'identità: la definizione dell'altro e del nemico. Il tema religioso ritorna decisivo e Lewis si concentra, per spiegare questi concetti, sullinterpretazione del Jihad e sulla legislazione relativa alla Dhimma, ovvero sulla differenza tra Dar-al Islam e Dar-al Harb e sulle relazioni intracomunitarie tra le genti del Libro: Cristiani, Ebrei e Musulmani.

Il saggio si conclude con un agile capitolo sulle "Aspirazioni” del Medio Oriente. Qui, oltre a venir ribadito il tema della diversità insita in tutte le espressioni mediorientali, si insite sul ruolo di possibile guida regionale della Turchia o dell'Iran, ma anche sulle ancora numerose difficoltà che il Medio Oriente dovrà affrontare nel suo lento e lungo processo di trasformazione da una realtà plurale e fluida, com'è tutt'ora, ad una maggiormente rispondente ai canoni etno-nazionalisti occidentali impostisi da diverso tempo nei discorsi politici di tutta la regione.

Significativa a questo proposito è la premonizione fatta da Ali Paşa, ministro degli esteri ottomano, nel 1862:

l'Italia, che è abitata da una sola razza che parla la stessa lingua e professa la stessa religione, incontra grandi difficoltà nel conquistare l'unificazione. E per il momento tutto quello che ha ottenuto è anarchia e disordine. Giudicate cosa accadrebbe in Turchia se fosse dato pieno sfogo a tutte le diverse aspirazioni nazionali. [...] Occorrerebbero cento anni e fiumi di sangue per ristabilire uno stato di cose sufficientemente stabile ${ }^{6}$.

\footnotetext{
${ }^{6}$ BAYSUN, M. Cavid (a cura di), «Ali Paşa'nın Fransızca bır mektubu» in Tarth Dergisi, 5/1953, p. 144; citato in LEWIS, Bernard, Le molte identità del Medio Oriente, Bologna, Il Mulino, 2011, p. 153.
} 
Come ricorda nel paragrafo conclusivo Lewis, infatti, sono passati più di cent'anni e il sangue continua ancora a scorrere nel Medio Oriente senza che si sia raggiunto "uno stato di cose sufficientemente stabile".

Tracciando un bilancio di quanto detto, il saggio di Bernard Lewis si pone e si impone all'interno del più recente panorama di studi sulla regione del Medio Oriente come una pietra angolare delle nuove teorie storiografiche che, superato l'orientalismo e l'eurocentrismo, cercano di analizzare dal punto di vista mediorientale il Medio Oriente. La scrittura agile e semplice e il grande numero di esempi, sempre significativi e precisi, rendono questo saggio appetibile sia per il grande pubblico - ancora troppo digiuno sulle problematiche di questa complessa parte del Mondo -, sia per un pubblico di esperti che riescono ad intravedere le profondità tematiche e sintattiche che Lewis spesso lumeggia solamente per non appesantire la sua esposizione. La precedente struttura di paper per un convegno emerge infatti in alcune parti del saggio, che potrebbero avere anche vita autonoma e approfondimenti molto più dettagliati. Unica pecca di questo pur ottimo lavoro, è la quasi totale mancanza di note e di bibliografia. Questa assenza, purtroppo, rende meno fruibile il saggio di Lewis dal momento che non permette a chi volesse avvicinarsi o approfondire gli argomenti trattati di risalire alle fonti impiegate.

Resta indubbio il grande lavoro di decodificazione operato dall'autore sui concetti e sulle principali idee che popolano i discorsi politici mediorientali contemporanei e che consentono, assieme alle chiare definizioni date da Lewis, di far maggiore chiarezza su temi, società e Paesi ancora in divenire, che rendono spesso difficile la comprensione delle vicende mediorientali. 


\section{* L'autore}

Luca Zuccolo, dottore (PhD) in Storia Contemporanea del SUM di Napoli ha sviluppato una ricerca sulle dinamiche di formazione dell'identità ottomana e di un discorso di tipo patriottico durante il regno di Abdülhamid II (1880-1885) attraverso lo studio di fonti a stampa redatte in lingua francese. Già dottore magistrale in Storia d'Europa (Bologna 2008), si è occupato dello sviluppo della modernità durante l'ultimo secolo dell'Impero Ottomano, del confronto/scontro tra modernità e tradizione in un contesto cosmopolita e allo sviluppo dei movimenti sociali che hanno preparato l'avvento della società turca contemporanea.

URL: <www.studistorici.com/2010/12/o1/luca-zuccolo >

\section{Per citare questo articolo:}

ZUCCOLO, Luca, «Recensione: Bernard LEWIS, Le molte identità del Medio Oriente, Bologna, il Mulino, 2011, 160 pp.», Diacronie. Studi di Storia Contemporanea: Sulle tracce delle idee, 29/12/2012,

URL:< http://www.studistorici.com/2012/12/29/zuccolo-lewis_numero_12/ >

Diacronie Studi di Storia Contemporanea www.diacronie.it

Risorsa digitale indipendente a carattere storiografico. Uscita trimestrale.

redazione.diacronie@hotmail.it

Comitato di redazione: Marco Abram - Jacopo Bassi - Luca Bufarale - Alessandro Cattunar - Alice De Rensis - Barbara Galimberti Deborah Paci - Fausto Pietrancosta - Matteo Tomasoni - Luca Zuccolo

Diritti: gli articoli di Diacronie. Studi di Storia Contemporanea sono pubblicati sotto licenza Creative Commons 2.5

Possono essere riprodotti a patto di non modificarne i contenuti e di non usarli per fini commerciali. La citazione di

estratti è comunque sempre autorizzata, nei limiti previsti dalla legge. 